\title{
Chronic kidney disease in children and the role of epigenetics: Future therapeutic trajectories (Review)
}

\author{
SAMUEL N. UWAEZUOKE ${ }^{1}$, HENRIETTA U. OKAFOR ${ }^{1}$, VIVIAN N. MUONEKE ${ }^{1}$, \\ ODUTOLA I. ODETUNDE ${ }^{1}$ and CHIOMA L. ODIMEGWU ${ }^{2}$ \\ ${ }^{1}$ Department of Pediatrics, College of Medicine; ${ }^{2}$ Department of Pediatrics, University \\ of Nigeria Teaching Hospital, Ituku-Ozalla, Enugu 400001, Nigeria
}

Received August 3, 2016; Accepted October 4, 2016

DOI: $10.3892 /$ br.2016.781

\begin{abstract}
Global differences in the observed causes of chronic kidney disease (CKD) in children are well documented and are attributed to dissimilarities in clime, race, hereditary, and ancestry. Thus, familial clustering and disparities in CKD prevalence rates across ethnic and racial groups indicate that the progression of renal disease has a strong genetic component. Mammalian studies have demonstrated a feasible nexus between nutrition and non-genetic exposure (around the time of conception and in epigenetic changes) in the expression of major genes identified in renal organogenesis. The major consequence is a reduction in the number of nephrons, with subsequent predisposition to hypertension and CKD. Identifying these epigenetic changes is crucial (due to their potentially reversible nature), as they may serve as future therapeutic targets to prevent kidney fibrosis and CKD. Despite progress in the field of epigenetics in oncology, research in other subspecialties of medicine is largely experimental with few existing studies regarding the clinical implication of epigenetics in renal disease. Therapeutic trajectories for CKD in children based on the influence of epigenetics may eventually revolutionize the management of this disease. The aim of the current narrative review is to appraise the role of epigenetics in CKD, and highlight the potential future therapeutic pathways.
\end{abstract}

\section{Contents}

1. Introduction

2. Epigenetics: Definition and pathophysiology

3. Role of epigenetics in CKD

4. Future therapeutic trajectories

5. Conclusion

Correspondence to: Dr Samuel N. Uwaezuoke, Department of Pediatrics, College of Medicine, University of Nigeria Teaching Hospital, Ituku-Ozalla, Enugu 400001, Nigeria

E-mail: snuwaezuoke@yahoo.com

Key words: epigenetics, chronic kidney disease, children, therapeutic pathways

\section{Introduction}

Chronic kidney disease (CKD) contributes significantly to the global health burden; it has an estimated prevalence of $8-16 \%$ worldwide and results in millions of mortalities annually, due to poor access to affordable treatment in resource-limited settings $(1,2)$. CKD is an important determinant of the poor health outcomes of major non-communicable diseases, and is a notable risk multiplier in patients with diabetes and hypertension (3). There is a paucity of data regarding the epidemiology of the early stages of pediatric CKD, as a result of under-diagnosis and under-reporting. However, statistics indicate that congenital disorders, including congenital anomalies of the kidney and urinary tract, and hereditary nephropathies, are responsible for approximately $2 / 3$ of all cases of CKD in developed countries, whereas acquired causes predominate in developing countries (4). There are very clear geographic diversities in the reported causes of pediatric CKD, which are attributed to dissimilarities in clime, race, hereditary and ancestry (5). Thus, familial clustering and disparities in CKD prevalence rates across ethnic and racial groups indicate that progression of renal disease has a strong genetic component (6-9).

Previous hereditary-associated studies have revealed common loci that may increase CKD risk (10). However, the majority of risk alleles for CKD identified by the genome-wide association studies (GWAS) confer only a very small relative risk. For example, the CKD Genetics study groups conducted a meta-analysis of GWAS data in 67,093 subjects of Caucasian descent from 20 predominantly demographic studies; the 16 analyzed loci accounted for only $1.4 \%$ of the variation in estimated glomerular filtration rate, indicating that other hereditary and non-genetic factors may be associated with CKD risk (10). According to this concept of 'missing heritability', epigenetic modifications presumably contribute to the inherited risk for CKD, as well as contribute to the explanation for the environmental influence on the human genome, which alters an individual's susceptibility to disease. Although there is no change in DNA sequence, modification in genome structure results in heritable and potentially reversible alterations in gene expression (11). Notably, mammalian studies have demonstrated a feasible link between nutrition and non-genetic exposure, around the time of conception, 
as well as in epigenetic changes, in the expression of major genes identified in renal organogenesis (11). The major consequence is the reduction in the number of nephrons, with subsequent predisposition to hypertension and CKD. Similarly, adverse intrauterine and postnatal conditions have a prolonged role in the evolution of CKD over the lifetime of an individual. Such adverse intrauterine conditions include small-for-gestational age infants or conversely, macrosomic infants of diabetic mothers. In addition, poor diabetes control $\geq 25$ years earlier leads to increased susceptibility to nephropathy regardless of a decade of excellent glycemic control (the 'metabolic memory' phenomenon). Identifying these epigenetic changes is crucial because of their potentially reversible nature; these epigenetic changes may serve as future therapeutic targets to prevent kidney fibrosis and CKD (11). Despite the increase in epigenetic research and the rising evidence of its relevance in the evolution of various complex disease traits, it is more prominent in oncology. Although the deviation into epigenetics by other subspecialties remains tentative, such research is largely experimental with few existing reports regarding the clinical implication of epigenetics in kidney disease (12). The aim of the current review was to appraise the role of epigenetics in CKD, and highlight potential future therapeutic pathways.

\section{Epigenetics: Definition and pathophysiology}

The term 'epigenetics' is best understood within the context of the interaction between the gene and the environment (Fig. 1). From a historical perspective, the definition of the term has metamorphosed over the years from its original coinage and meaning by Slack (13). More than 10 years later, another author defined 'epigenetic systems' as supportive processes involved in ascertaining the specific genes that will be expressed in any individual cell (14). Following the identification of heritable models of DNA methylation, the hypothesis that epigenetic characteristics were inherited as controlling indicators coupled with the genome predominated. Epigenetics was subsequently defined as the investigation of mitotically and/or meiotically inheritable alterations in gene expression, which are not associated with alterations in DNA sequence (15). The identification of the regulatory function of histone post-translational modifications and their reciprocal association with transcriptional states encouraged a more relaxed use of the term 'epigenetics', to refer to any molecular imprint located on chromosomes (particularly histone marks) and loosely defined as the configurational adjustment of chromosomal loci in order to express, indicate or prolong altered activity (16). Simultaneously, a narrower definition was suggested as a consistently inheritable phenotype due to changes in a chromosome, which are not associated with alterations in the DNA sequence (17). Subsequently, epigenetics came to be defined as the inheritance of variation (-genetics) above and beyond (epi-) alterations in the DNA sequence (18). Therefore, three main epigenetic mechanisms are recognized in mammals as follows: Changes in DNA methylation, histone post-translational modifications and RNA interference (non-coding RNAs) (19). DNA methylation is an important epigenetic factor that is responsible for the control of gene expression and genomic consistency, and is biologically vital for the preservation of various physiologic activities of the cell (20).
The epigenome is the interconnecting point of genetics and environment, which stimulates changes that modulate disease phenotype by direct effect on the target gene, irrespective of changes in sequence within the gene (Fig. 2). Possible triggers include environmental signals and pathologic states, such as diet, exercise, inflammation, oxidative stress, metabolic changes and toxins (21-23). Epigenetic changes are potentially reversible $(24,25)$; notably, to simple measures, such as lifestyle modifications and nutrient supplementation (26-29).

Regarding the pathophysiology of epigenetic processes, DNA methylation occurs at the 5-cystine of $\mathrm{CpG}$ dinucleotides. Briefly, hypermethylation switches off the gene expression (gene silencing), while hypomethylation switches it on or leads to the re-expression of a normally silenced gene. Hypomethylation and genomic disequilibrium results from imbalances in dietary nutrients, such as folate (29). Methyl groups originating from the diet are passed on to DNA via folate and methionine pathways, and 5-methyl cytosine content is modified by the nutritional accessibility to folate (19). Furthermore, infections (particularly viral infections) initiate DNA methylation. Thus, variations in DNA-methylation patterns occur following dietary changes, inherited genetic polymorphisms and exposure to environmental factors.

Additionally, with respect to histone modification, DNA is transcriptionally active when histones are acetylated and unmethylated, whereas deacetylated and methylated histones block gene expression (30). Histone methylation occurs on Arg or Lys residues, with a discriminating site selectivity for Lys methylation at specific positions in the $\mathrm{N}$-end points of histones $\mathrm{H} 3$ and H4. Thus, Lys methylation is added to acetylation and phosphorylation as a third component of a 'histone code', which alters the fundamental chromatin makeup of the genome. The adjustment of this 'histone code' influences the maintenance of gene expression with inheritable epigenetic patterns (31).

Finally, cleavage and degradation of microRNA (miRNA) block translational mechanisms and prevent protein formation, resulting in silencing of gene expression. Double-stranded RNAs also cause transcriptional silencing via methylation of homologous DNA promoter sequences (32) and/or formation of heterochromatin (33). This RNA interference is vital for the evolution of kidney disease (12), as miRNAs are critical in the preservation of glomerular balance (34).

\section{Role of epigenetics in CKD}

A recent review highlights various evidence-based studies, which show the association between the intrauterine environment/number of nephrons, and the development of nephropathy (35). For example, epidemiologic data has revealed an association between low birth weight and subsequent non-communicable diseases, such as adult hypertension, diabetes, cardiovascular disease and CKD (36). Furthermore, a direct correlation between low birth weight and microalbuminuria has been reported in insulin-dependent diabetes mellitus (IDDM), as well as in older non-diabetic subjects (37). Low birth weight also directly correlates with albuminuria in non-IDDM and with the progression of CKD (37). Notably, findings from animal and human studies support the hypothesis that low 


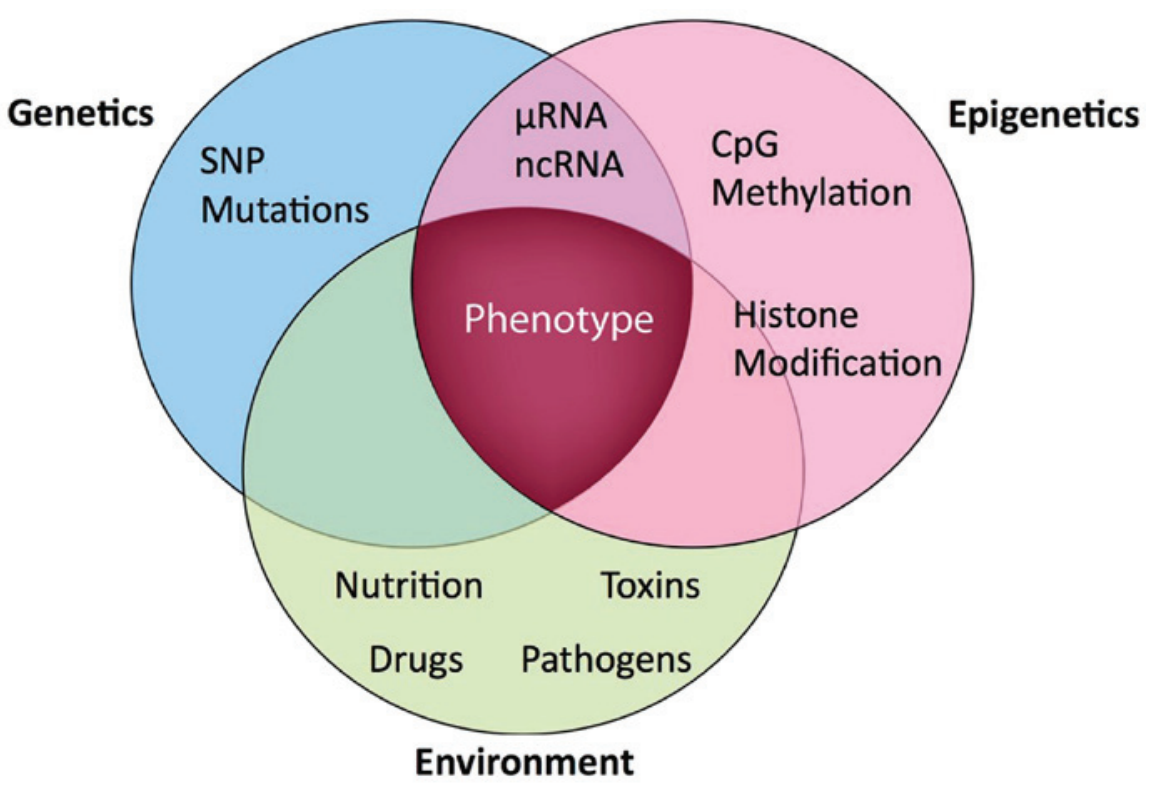

Figure 1. Schematic diagram representing the gene-environment interaction (genetic-epigenetic phenotype) (12). SNP, single nucleotide polymorphism; ncRNA, non-coding RNA.

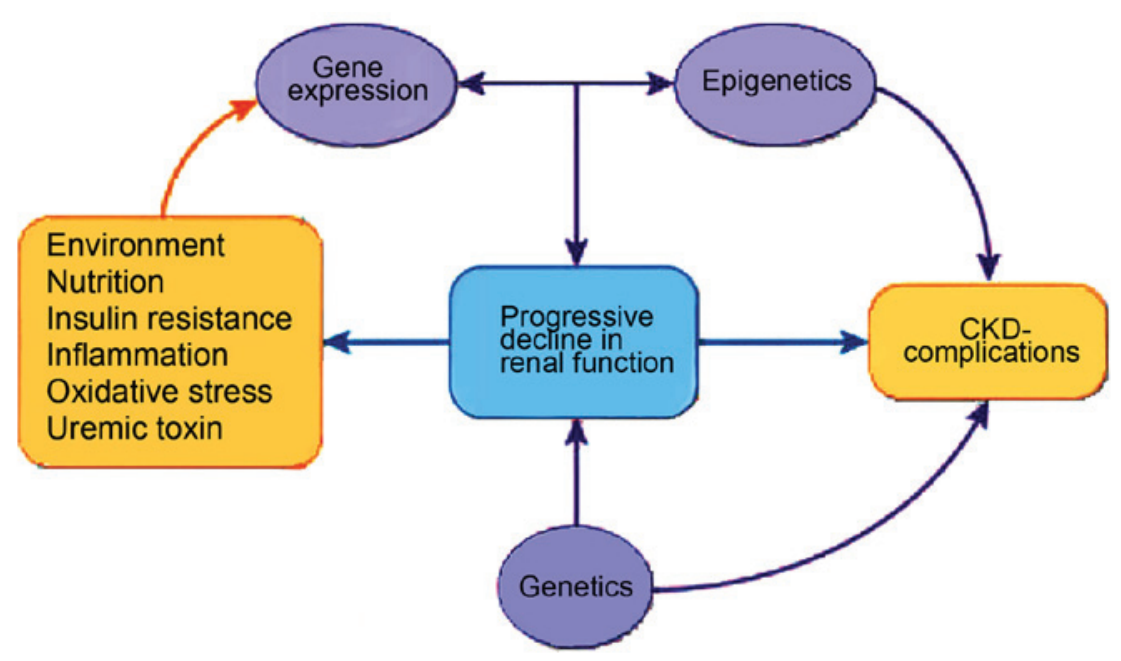

Figure 2. CKD phenotype and epigenetics (12). CKD, chronic kidney disease.

birth weight (a reflection of an unfavorable intrauterine environment) is accompanied by a reduction in the number of nephrons at birth (37). In man, the ultimate acquisition of nephrons depends on the intrauterine milieu and gestational age at delivery, as post-natal nephrogenesis is absent. Growth-retarded stillbirths and live-born infants with intrauterine growth retardation demonstrated a diminished number of nephrons when compared with control infants whose birth weights were appropriate for their gestational age (38). However, the caveat is that normal birth weight is not often synonymous with a conventional number of nephrons. To further support these observations, autopsy studies in adults indicated a significant correlation between birth weights/number of nephrons, and mean arterial pressure (39). A previous study described a case series $(n=6)$ of focal segmental glomerulosclerosis (FSGS) where the clinicopathologic results strongly agreed with the secondary form of FSGS, in which the only observed risk factors were prematurity and very low birth weight (40).

There is substantial evidence showing the role of key epigenetic mechanisms in the progression of CKD. A previous study observed a difference in DNA methylation patterns identified in renal fibroblasts between normal and diseased kidneys (41); DNA methylation may regulate fibroblast multiplication and contribute to renal fibrogenesis. For example, in the study, experimental murine models treated with 5-azacytidine, in which DNA methylation was impeded, experienced protection from kidney fibrosis. The observation further underscores the fact that hypermethylation contributes to renal fibrogenesis, and is the first report to show that DNA methylation directly affects the evolution of CKD. In addition, these investigators also demonstrated that hypermethylation of RAS protein activator like-1 was associated with the sustenance of fibroblast proliferation and renal fibrogenesis (41). 
Although the various histone modifications in the human kidney have not been evaluated with respect to CKD, studies conducted in various murine models of nephropathy indicate major disparities in the 'histone code' (35). Certain studies demonstrated that elevated levels of renal H3K9 and H3K23 acetylation, $\mathrm{H} 3 \mathrm{~K} 4$ dimethylation and $\mathrm{H} 3$ phosphorylation at serine 10 were noted in murine models of advanced diabetic nephropathy $(42,43)$. Furthermore, there are experimental instances showing the effect of posttranslational histone modifications, which induce renin cell identity. Using a cultured renin linage cell system, studies observed that its gene expression memory is maintained in the cultured cells and may be re-enacted by CAMP and chromatin remodeling (histone H4 acetylation) (44). Other investigators also noted that CREB-binding protein and p300 (which possess histone acetyltransferase activity) are vital for renin cell distinctiveness, as well as the integrity of the renal architecture (45).

Finally, the evidence for the role of RNA interference in CKD includes the following: First, one study demonstrated that after dicer (miRNA-generating enzyme) was inactivated in murine podocytes, the experimental mice presented with proteinuria and subsequently succumbed due to renal failure (46). The glomeruli have demonstrated effacement of the foot process, podocyte cell death, expansion of the mesangium and glomerulosclerosis (47). Additionally, previous studies have identified that disruption of miRNA biogenesis in murine podocytes was followed by proteinuria, podocyte dedifferentiation and crescent structure resulting in end-stage renal disease $(\mathrm{ESRD})(48,49)$.

\section{Future therapeutic trajectories}

Control of the epigenetic gene is hypothetically compliant to interventional measures given the non-mutation of the gene by methylation and the fact that the chromatin is not irreversibly changed (12). An interventional study evaluated the outcome of folate administration on DNA methylation in ESRD subjects with hyperhomocysteinaemia (50); the preliminary findings support a previous observation that hyperhomocysteinaemia-induced DNA hypomethylation could be reversed by administration of folate (51).

Notably, numerous epigenetic therapeutic agents are in various developmental phases despite their limitation in target specificity (52). Certain epigenetic therapeutic agents include nucleoside and non-nucleoside analogs, which are also known as inhibitors of DNA methylation (52). For example, myelodysplastic syndrome and secondary acute myeloid leukemia, which are unresponsive to conventional chemotherapy, have been managed with DNA methyltransferase inhibitors, including 5-azacytidine and decitabine with favorable outcomes (53).

Furthermore, inhibitors of histone deacytylase, which consist of a large, heterogeneous group of therapeutic agents comprising short-chain fatty acids, hydroxamic acids, benzamides and cyclic tetrapeptides are also within the future treatment trajectory for CKD. Specifically, all trans-retinoic acids and 13-cis-retinoic acid are triggers of hyperacetylation. In addition, they are being used experimentally in the treatment of pauci-immune vasculitis (12). On a theoretical basis, small interfering RNA may be employed to treat any disease arising from overexpression of a distinctive gene. Therefore, this technique might be applicable to genes implicated in inflammation, proliferation and fibrosis. In fact, nucleic acid-based interventions are currently being performed for atherosclerosis and progressive renal diseases (54). As there are more breakthroughs regarding the role of epigenetics in CKD, it is hoped that novel markers for diagnostic purposes, as well as treatment-driven molecular pathways will continue to emerge in the field of renal medicine.

\section{Conclusion}

Advances in comprehending epigenetic alterations in the evolution of CKDs may significantly predict the speed of disease progression, develop targeted treatment strategies in preventing its progression, and provide an efficient management of uremia-associated complications (12). The current limitation of epigenetic therapeutic agents, which remain largely in the developmental stage, is their lack of target specificity. Furthermore, there are ongoing efforts to conduct gene expression studies and epigenomics analysis to identify novel diagnostic and prognostic markers for progressive renal diseases. Therapeutic trajectories for CKD in children based on the influence of epigenetics may in future revolutionize the management of CKD.

\section{References}

1. World Kidney Day: Chronic Kidney Disease. 2015; http://www. worldkidneyday.org/faqs/chronic-kidney-disease/. Accessed October 10, 2014.

2. Jha V, Garcia-Garcia G, Iseki K, Li Z, Naicker S, Plattner B, Saran R, Wang AY and Yang CW: Chronic kidney disease: Global dimension and perspectives. Lancet 382: 260-272, 2013.

3. Couser WG, Remuzzi G, Mendis S and Tonelli M: The contribution of chronic kidney disease to the global burden of major noncommunicable diseases. Kidney Int 80: 1258-1270, 2011.

4. Harambat J, van Stralen KJ, Kim JJ and Tizard EJ: Epidemiology of chronic kidney disease in children. Pediatr Nephrol 27: 363-373, 2012.

5. Warady BA and Chadha V: Chronic kidney disease in children: The global perspective. Pediatr Nephrol 22: 1999-2009, 2007.

6. Bowden DW: Genetics of kidney disease. Kidney Int Suppl 83: S8-S12, 2003.

7. Lei HH, Perneger TV, Klag MJ, Whelton PK and Coresh J: Familial aggregation of renal disease in a population-based case-control study. J Am Soc Nephrol 9: 1270-1276, 1998.

8. Hsu CY, Lin F, Vittinghoff E and Shlipak MG: Racial differences in the progression from chronic renal insufficiency to end-stage renal disease in the United States. J Am Soc Nephrol 14: 2902-2907, 2003.

9. Freedman BI, Spray BJ, Tuttle AB and Buckalew VM Jr: The familial risk of end-stage renal disease in African Americans. Am J Kidney Dis 21: 387-393, 1993.

10. Köttgen A, Pattaro C, Böger CA, Fuchsberger C, Olden M, Glazer NL, Parsa A, Gao X, Yang Q, Smith AV, et al: New loci associated with kidney function and chronic kidney disease. Nat Genet 42: 376-384, 2010.

11. Liakopoulos V, Georgianos PI, Eleftheriadis T and Sarafidis PA: Epigenetic mechanisms and kidney diseases. Curr Med Chem 18: 1733-1739, 2011.

12. Dwivedi RS, Herman JG, McCaffrey TA and Raj DSC: Beyond genetics: Epigenetic code in chronic kidney disease. Kidney Int 79: 23-32, 2011.

13. Slack JM: Conrad Hal Waddington: The last Renaissance biologist? Nat Rev Genet 3: 889-895, 2002.

14. Nanney DL: Epigenetic control systems. Proc Natl Acad Sci USA 44: 712-717, 1958 .

15. Riggs A, Martienssen R and Russo V (eds): Epigenetic mechanisms of gene regulation. Vol. 32. Cold Spring Harbor Laboratory Press, 1996. 
16. Bird A: Perceptions of epigenetics. Nature 447: 396-398, 2007.

17. Berger SL, Kouzarides T, Shiekhattar R and Shilatifard A: An operational definition of epigenetics. Genes Dev 23: 781-783, 2009.

18. Bonasio R, Tu S and Reinberg D: Molecular signals of epigenetic states. Science 330: 612-616, 2010.

19. Link A, Balaguer F and Goel A: Cancer chemoprevention by dietary polyphenols: Promising role for epigenetics. Biochem Pharmacol 80: 1771-1792, 2010.

20. Sadikovic B, Al-Romaih K, Squire JA and Zielenska M: Cause and consequences of genetic and epigenetic alterations in human cancer. Curr Genomics 9: 394-408, 2008.

21. Luch A: Nature and nurture-lessons from chemical carcinogenesis. Nat Rev Cancer 5: 113-125, 2005.

22. Perna AF, Ingrosso D, Galletti P, Zappia V and De Santo NG: Membrane protein damage and methylation reactions in chronic renal failure. Kidney Int 50: 358-366, 1996.

23. Robertson KD and Wolffe AP: DNA methylation in health and disease. Nat Rev Genet 1: 11-19, 2000

24. Ingrosso D, Cimmino A, Perna AF, Masella L, De Santo NG, De Bonis ML, Vacca M, D'Esposito M, D'Urso M, Galletti P, et al: Folate treatment and unbalanced methylation and changes of allelic expression induced by hyperhomocysteinaemia in patients with uraemia. Lancet 361: 1693-1699, 2003.

25. Ptak C and Petronis A: Epigenetics and complex disease: From etiology to new therapeutics. Annu Rev Pharmacol Toxicol 48: 257-276, 2008

26. Huang Y, Chang X, Lee J, Cho YG, Zhong X, Park IS, Liu JW, Califano JA, Ratovitski EA, Sidransky D, et al: Cigarette smoke induces promoter methylation of single-stranded DNA-binding protein 2 in human esophageal squamous cell carcinoma. Int J Cancer 128: 2261-2273, 2011.

27. Nakajima K, Takeoka M, Mori M, Hashimoto S, Sakurai A, Nose H, Higuchi K, Itano N, Shiohara M, Oh T, et al: Exercise effects on methylation of ASC gene. Int J Sports Med 31: 671-675, 2010.

28. Oommen AM, Griffin JB, Sarath G and Zempleni J: Roles for nutrients in epigenetic events. J Nutr Biochem 16: 74-77, 2005.

29. Duthie SJ: Folic acid deficiency and cancer: Mechanisms of DNA instability. Br Med Bull 55: 578-592, 1999.

30. Peterson CL and Laniel MA: Histones and histone modifications. Curr Biol 14: R546-R551, 2004.

31. Jenuwein T: Re-SET-ting heterochromatin by histone methyltransferases. Trends Cell Biol 11: 266-273, 2001.

32. Tang W, Luo XY and Sanmuels V: Gene silencing: Double-stranded RNA mediated mRNA degradation and gene inactivation. Cell Res 11: 181-186, 2001.

33. Wassenegger M: The role of the RNAi machinery in heterochromatin formation. Cell 122: 13-16, 2005.

34. Schena FP, Serino G and Sallustio F: MicroRNAs in kidney diseases: New promising biomarkers for diagnosis and monitoring. Nephrol Dial Transplant 29: 755-763, 2014.

35. Woroniecki R, Gaikwad AB and Susztak K: Fetal environment, epigenetics, and pediatric renal disease. Pediatr Nephrol 26: 705-711, 2011

36. Zandi-Nejad K, Luyckx VA and Brenner BM: Adult hypertension and kidney disease: The role of fetal programming. Hypertension 47: 502-508, 2006.

37. Luyckx VA and Brenner BM: Low birth weight, nephron number, and kidney disease. Kidney Int Suppl 68: S68-S77, 2005.

38. Alexander BT: Intrauterine growth restriction and reduced glomerular number: Role of apoptosis. Am J Physiol Regul Integr Comp Physiol 285: R933-R934, 2003.
39. Hughson MD, Douglas-Denton R, Bertram JF and Hoy WE: Hypertension, glomerular number, and birth weight in African Americans and white subjects in the southeastern United States. Kidney Int 69: 671-678, 2006.

40. Hodgin JB, Rasoulpour M, Markowitz GS and D'Agati VD: Very low birth weight is a risk factor for secondary focal segmental glomerulosclerosis. Clin J Am Soc Nephrol 4: 71-76, 2009.

41. Bechtel W, McGoohan S, Zeisberg EM, Müller GA, Kalbacher H, Salant DJ, Müller CA, Kalluri R and Zeisberg M: Methylation determines fibroblast activation and fibrogenesis in the kidney. Nat Med 16: 544-550, 2010

42. Sayyed SG, Gaikwad AB, Lichtnekert J, Kulkarni O, Eulberg D, Klussmann S, Tikoo K and Anders HJ: Progressive glomerulosclerosis in type 2 diabetes is associated with renal histone $\mathrm{H} 3 \mathrm{~K} 9$ and $\mathrm{H} 3 \mathrm{~K} 23$ acetylation, $\mathrm{H} 3 \mathrm{~K} 4$ dimethylation and $\beta$ phosphorylation at serine 10. Nephrol Dial Transplant 25: 1811-1817, 2010.

43. Gaikwad AB, Sayyed SG, Lichtnekert J, Tikoo K and Anders HJ: Renal failure increases cardiac histone h3 acetylation, dimethylation, and phosphorylation and the induction of cardiomyopathy-related genes in type 2 diabetes. Am J Pathol 176: 1079-1083, 2010.

44. Pentz ES, Lopez ML, Kim HS, Carretero O, Smithies O and Gomez RA: Ren1d and Ren2 cooperate to preserve homeostasis: Evidence from mice expressing GFP in place of Ren1d. Physiol Genomics 6: 45-55, 2001.

45. Gomez RA, Pentz ES, Jin X, Cordaillat $M$ and Sequeira Lopez ML: CBP and p300 are essential for renin cell identity and morphological integrity of the kidney. Am J Physiol Heart Circ Physiol 296: H1255-H1262, 2009.

46. Shi S, Yu L, Chiu C, Sun Y, Chen J, Khitrov G, Merkenschlager M, Holzman LB, Zhang W, Mundel P, et al: Podocyte-selective deletion of dicer induces proteinuria and glomerulosclerosis. J Am Soc Nephrol 19: 2159-2169, 2008.

47. Ho JJ and Marsden PA: Dicer cuts the kidney. J Am Soc Nephrol 19: 2043-2046, 2008.

48. Harvey SJ, Jarad G, Cunningham J, Goldberg S, Schermer B, Harfe BD, McManus MT, Benzing T and Miner JH: Podocyte-specific deletion of dicer alters cytoskeletal dynamics and causes glomerular disease. J Am Soc Nephrol 19: 2150-2158, 2008.

49. Ho J, Ng KH, Rosen S, Dostal A, Gregory RI and Kreidberg JA: Podocyte-specific loss of functional microRNAs leads to rapid glomerular and tubular injury. J Am Soc Nephrol 19: 2069-2075, 2008 .

50. Ingrosso D, Cimmino A, Perna AF, Masella L, De Santo NG, De Bonis ML, Vacca M, D'Esposito M, D'Urso M, Galletti P, et al: Folate treatment and unbalanced methylation and changes of allelic expression induced by hyperhomocysteinaemia in patients with uraemia. Lancet 361: 1693-1699, 2003.

51. Newman PE: Can reduced folic acid and vitamin B12 levels cause deficient DNA methylation producing mutations which initiate atherosclerosis? Med Hypotheses 53: 421-424, 1999.

52. Ptak $\mathrm{C}$ and Petronis A: Epigenetics and complex disease: From etiology to new therapeutics. Annu Rev Pharmacol Toxicol 48: 257-276, 2008

53. Griffiths EA and Gore SD: DNA methyltransferase and histone deacetylase inhibitors in the treatment of myelodysplastic syndromes. Semin Hematol 45: 23-30, 2008.

54. Fukuda N: Development of gene therapies for cardiovascular and renal diseases by nucleic acid medicines. Med Chem 2: 13-19, 2006. 